\title{
Baseline Characteristics and Outcomes of End-Stage Renal Disease Patients after In-hospital Sudden Cardiac Arrest: A National Perspective
}

\author{
Muhammad Khan ${ }^{1}$, Moinuddin Syed ${ }^{2}$, Pratik Agrawal ${ }^{1}$, Mohammed Osman ${ }^{1}$, Muhammad \\ Khan $^{1}$, Anas Alharbi ${ }^{1}$, Mina Benjamin ${ }^{2}$, Sudarshan Balla ${ }^{1}$, and Muhammad Bilal Munir ${ }^{2}$ \\ ${ }^{1}$ West Virginia University Hospitals \\ ${ }^{2}$ West Virginia University School of Medicine
}

April 27, 2020

\begin{abstract}
Background: End stage renal disease (ESRD) is a well-recognized risk factor for development of sudden cardiac arrest (SCA). There is limited data on baseline characteristics and outcomes after an in-hospital SCA event in ESRD patients. Methods: For the purpose of this study, data were obtained from National Inpatient Sample from January 2007 to December 2017. In-hospital SCA was identified using International Classification of Disease, 9th Revision, Clinical Modification and International Classification of Disease, 10th Revision, Clinical Modification codes of 99.60, 99.63 and 5A12012. ESRD patients were subsequently identified using codes of 585.6 and N18.6. Baseline characteristics and outcomes were compared among ESRD and non-ESRD patients in crude and propensity matched (PS) cohorts. Predictors of mortality in ESRD patients after an in-hospital SCA event were analyzed using a multivariate logistic regression model. Results: A total of 1,412,985 patients sustained in-hospital SCA during our study period. ESRD patients with in-hospital SCA were younger and had a higher burden of key co-morbidities. Mortality was similar in ESRD and non-ESRD patients in PS matched cohort $(70.4 \%$ vs. $70.7 \%, \mathrm{p}=0.45)$ with an overall downward trend over our study years. Advanced age, Black race, diabetes, hypertension and peripheral vascular disease were independently associated with increased mortality in ESRD patients after an in-hospital SCA event. Conclusion: In the context of in-hospital SCA, mortality is similar in ESRD and non-ESRD patients in adjusted analysis. Adequate risk factor modification could further mitigate the risk of in-hospital SCA among ESRD patients.
\end{abstract}

\section{Baseline Characteristics and Outcomes of End-Stage Renal Disease Patients after In-hospital Sudden Cardiac Arrest: A National Perspective}

Muhammad Zia Khan, MD ${ }^{1}$, Moinuddin Syed, MD ${ }^{1}$, Pratik Agrawal, MD ${ }^{1}$, Mohammed Osman, MD ${ }^{1}$, Muhammad U. Khan, MD ${ }^{1}$, Anas Alharbi, MD ${ }^{1}$, Mina M. Benjamin, MD ${ }^{1}$, Sudarshan Balla, MD ${ }^{1}$, Muhammad Bilal Munir, MD ${ }^{1,2}$

${ }^{1}$ Division of Cardiovascular Medicine, West Virginia University Heart and Vascular Institute, Morgantown, WV, USA

${ }^{2}$ Section of Electrophysiology, Division of Cardiovascular Medicine, University of California San Diego, La Jolla, CA, USA

\section{Corresponding Author:}

Muhammad Bilal Munir, MD

West Virginia University Heart \& Vascular Institute and University of California San Diego Sulpizio Cardiovascular Center 
1 Medical Drive, Morgantown, West Virginia 26505

Phone: 304-598-4687

Fax: 304-599-0860

Email: muhamad.munir@hsc.wvu.edu

Disclosures: none

Funding: none

Word count: 2337

\section{Abstract: \\ Background:}

End stage renal disease (ESRD) is a well-recognized risk factor for development of sudden cardiac arrest (SCA). There is limited data on baseline characteristics and outcomes after an in-hospital SCA event in ESRD patients.

\section{Methods:}

For the purpose of this study, data were obtained from National Inpatient Sample from January 2007 to December 2017. In-hospital SCA was identified using International Classification of Disease, 9th Revision, Clinical Modification and International Classification of Disease, 10th Revision, Clinical Modification codes of 99.60, 99.63 and 5A12012. ESRD patients were subsequently identified using codes of 585.6 and N18.6. Baseline characteristics and outcomes were compared among ESRD and non-ESRD patients in crude and propensity matched (PS) cohorts. Predictors of mortality in ESRD patients after an in-hospital SCA event were analyzed using a multivariate logistic regression model.

\section{Results:}

A total of 1,412,985 patients sustained in-hospital SCA during our study period. ESRD patients with inhospital SCA were younger and had a higher burden of key co-morbidities. Mortality was similar in ESRD and non-ESRD patients in PS matched cohort $(70.4 \%$ vs. $70.7 \%, \mathrm{p}=0.45)$ with an overall downward trend over our study years. Advanced age, Black race, diabetes, hypertension and peripheral vascular disease were independently associated with increased mortality in ESRD patients after an in-hospital SCA event.

\section{Conclusion:}

In the context of in-hospital SCA, mortality is similar in ESRD and non-ESRD patients in adjusted analysis. Adequate risk factor modification could further mitigate the risk of in-hospital SCA among ESRD patients.

Key words : End-stage renal disease; In-hospital cardiac arrest; Outcome; Trends

\section{Introduction:}

Sudden cardiac arrest (SCA) is a prevalent entity in patients with end stage renal disease (ESRD). Nearly one quarter of deaths in this patient population is attributed to SCA (1). The mortality rate after a SCA event exceeds $52 \%$ in ESRD patients (2). ESRD patients are uniquely prone to the development of SCA. Majority of these patients have left ventricular hypertrophy $(\mathrm{LVH})$ which provides an underlying substrate for SCA perpetuation in settings of rapid fluid and electrolyte fluctuations during dialysis sessions $(3,4,5,6)$. ESRD patients also require frequent hospitalizations due to associated co-morbid conditions (7). Recent evidence points to improved outcomes in patients with in-hospital SCA over the past two decades (8). Limited data, however, exit in the context of ESRD patients after in-hospital SCA and whether these improved outcomes have also been witnessed in this patient population are currently unknown. In this paper, we aimed to study baseline characteristics, trends and outcomes of ESRD patients after they sustained in-hospital SCA from a nationally representative contemporary cohort of US population. 


\section{Methods:}

Data from the National Inpatient Sample (NIS) were used for this study. NIS database has been made possible through sponsorship of federal agency for Healthcare Research and Quality (AHRQ). The main purpose of AHRQ is to enhance the quality, appropriateness, and effectiveness of health care services (9). NIS is publicly available all-payer administrative claims-based database. National estimates of the entire US hospitalized population were calculated using the Agency for Healthcare Research and Quality sampling and weighting method. Institutional review board approval was not required for this study given the de-identified nature of the NIS and its public availability.

We analyzed the NIS data from January 2007 to December 2017 using the International Classification of Disease, 9th Revision, Clinical Modification (ICD-9-CM) and International Classification of Disease, 10th Revision, Clinical Modification (ICD-10-CM) codes. Patients who sustained in-hospital SCA were identified by applying ICD-9-CM and ICD-10-CM codes of 99.60, 99.63 and 5A12012, respectively to any procedure field. These codes indicate utilization of cardiopulmonary resuscitation (CPR) and well representative of inhospital SCA from administrative datasets as shown by the earlier studies $(10,11)$. ESRD patients were then subsequently identified using ICD-9-CM and ICD-10-CM codes of 585.6 and N18.6, respectively. Patients were excluded if they were less than 18 years of age or had acute kidney injury (AKI) and prior history of renal transplantation. Baseline characteristics and outcomes were compared in ESRD patients who sustained in-hospital SCA to non-ESRD patients with in-hospital SCA. Propensity score matching was also done to balance confounding variables and outcomes were again assessed in both groups. Trends in in-patient mortality and length of stay (LOS) were also assessed. Predictors of in-patient mortality in ESRD patients after a SCA event were also analyzed.

Age, race, median income, urban/rural hospital, US region and Elixhauser comorbidities were selected for analysis. Descriptive statistics were presented as frequencies with percentages for categorical variables and as means with standard deviations or median with interquartile range as appropriate for continuous variables. Baseline characteristics were compared using a Pearson?2 test for categorical variables and independent samples t-test or non-parametric tests for continuous variables as appropriate. Median LOS, median cost of stay and mortality were calculated. The median cost of stay was adjusted for inflation (in comparison to December 2017). Simple linear regression or Chi-Square test was used for trend analysis over the study years as appropriate. To mitigate the risk of confounding and selection bias, a nearest neighbor 1:1 propensity score (PS) matching was done using a caliper width of 0.2. In this way, ESRD and non-ESRD patients were well matched with respect to demographic variables as shown in table 1. Predictors of mortality in ESRD patients who sustained in-hospital SCA were analyzed using a logistic regression model. A forward stepwise entry model was used for this purpose. Initially, all variables, which were significantly associated with mortality with a $\mathrm{p}$ value of less than 0.05 in univariate analysis, were entered in the model from the baseline table. Subsequently only those variables are retained in the model which were associated with mortality with a $\mathrm{p}$ value of less than 0.10 during forward entry. A type I error of less than 0.05 was considered statistically significant. All statistical analyses were performed using statistical package for social science (SPSS) version 26 (IBM Corp) and $\mathrm{R}$ version 3.5.

\section{Results:}

We identified a total of 1,412,985 patients from January 2007 to December 2017 who suffered in-hospital SCA after excluding for age [?] 18 years, AKI and prior history of renal transplantation. Out of these patients, about 123,962 (9.6\%) patients had ESRD (please see figure 1). Baseline characteristics of the study population are shown in table 1. ESRD patients who suffered in-hospital SCA were younger when compared to non-ESRD patients with in-hospital SCA (65 years vs. 69 years, $\mathrm{p}<0.01$ ). Female patients were equally represented in both groups ( $44.7 \%$ vs. $44.7 \%, \mathrm{p}=0.45)$. ESRD was less prevalent in White patients $(40.1 \%$ vs. $66.1 \%, \mathrm{p}<0.01)$ and more prevalent in Blacks $(36.7 \%$ vs. $18.6 \%, \mathrm{p}<0.01)$ and Hispanics $(15 \%$ vs. $9.1 \%, \mathrm{p}<0.01)$. In terms of co-morbidity burden, congestive heart failure $(34.8 \%$ vs. $21.8 \%, \mathrm{p}<0.01)$, complicated diabetes $(32.5 \%$ vs. $8.4 \%, \mathrm{p}<0.01)$, hypertension $(80.9 \%$ vs. $50.4 \%, \mathrm{p}<0.01)$, coronary artery disease $(39.4 \%$ vs. $30.8 \%, \mathrm{p}<0.01)$ and peripheral vascular disease $(19.9 \%$ vs. $9.9 \%, \mathrm{p}<0.01)$ were more 
prevalent in ESRD patients who sustained in-hospital SCA when compared to non-ESRD patients.

Crude and propensity matched outcomes are shown in table 2. A total of 1,035,037 (73.2\%) patients died in our cohort after sustaining an in-hospital SCA. No difference in mortality was noted in PS matched analysis among ESRD and non-ESRD patients with in-hospital SCA (70.4\% vs. $70.7 \%, \mathrm{p}=0.45)$. The median LOS was 7 days (range 2-15) among ESRD patients who survived in-hospital SCA when compared to 6 days (range 2-13) in non-ESRD patients. Overall cost of hospitalization was $80,150.5 \$$ (range $35,009 \$-177,894 \$$ ) in ESRD patients with in-hospital SCA when compared to $65,297 \$$ (range $28,195 \$-145,639 \$$ ) in non-ESRD patients. The utilization of invasive cardiovascular procedures such as diagnostic coronary angiogram $(7.8 \%$ vs. $10.6 \%, \mathrm{p}<0.01)$, percutaneous coronary intervention $(2.6 \%$ vs. $4.6 \%, \mathrm{p}<0.01)$ and intra-aortic balloon pump implantation $(1.7 \%$ vs. $3.4 \%, \mathrm{p}<0.01)$ were lower in ESRD patients when compared to non-ESRD patients.

Over our study period from 2007-2017, the proportion of ESRD and non-ESRD patients who sustained in-hospital SCA was similar (please see figure 2). In-patient mortality showed a downward trend for both ESRD and non-ESRD patients with in-hospital SCA after an initial spike in year 2009 (please see figure 3). Median LOS showed a stable trend over our study years (please see figure 4).

Predictors of mortality in ESRD patients after they sustained SCA are shown in figure 5. Advanced age (OR 1.02 per year increase, $95 \%$ CI 1.019-1.022), Black race (OR 1.127, 95\% CI 1.091-1.164), chronic pulmonary disease (OR 1.063, 95\% CI 1.029-1.098), coagulopathy (OR 1.129, 95\% CI 1.092-1.167), diabetes (OR 1.046, 95\% CI 1.009-1.084), hypertension (OR 1.06, 95\% CI 1.025-1.096), chronic liver disease (OR 1.308, 95\% CI 1.239-1.382) and peripheral vascular disease (OR 1.079, 95\% CI 1.044-1.115) were independently associated with increased mortality in ESRD patients after a SCA event.

\section{Discussion:}

In this investigation of in-hospital SCA patients stratified on the basis of ESRD status or not, we report several key findings. (1) The mortality in patients with in-hospital SCA were similar in PS matched analysis regardless of ESRD status (70.4\% vs. $70.7 \%, \mathrm{p}=0.45$ ). (2) Over the study period from 2007-2017, there was a reduced trend of mortality after a SCA event in both ESRD and non-ESRD patients after an initial spike in the year 2009. (3) ESRD patients who suffered in-hospital SCA were younger and had a higher burden of key co-morbidities when compared to non-ESRD patients with in-hospital SCA. (4) The utilization of invasive procedures was lower in ESRD patients compared to non-ESRD patients after an in-hospital SCA.

In-hospital SCA affects nearly 290,000 adult patients in US each year (12). The clinical trajectory of ESRD patients is frequently complicated by SCA which contributes to nearly a quarter of deaths in this patient population (1). ESRD patients have an underlying vulnerable myocardial substrate for SCA since most of these patients are found to have LVH that can prolong ventricular repolarization, a well-recognized risk factor for induction of malignant arrhythmias $(3,4)$. Additionally, electrolyte fluctuations during dialysis sessions are responsible for triggering a SCA event $(5,6)$. Few earlier studies have reported outcomes of ESRD patients after they sustained in-hospital SCA. In a previous study on outcomes in ESRD patients after in-hospital SCA, Saeed et al. have shown higher adjusted mortality in ESRD patients when compared to non-ESRD group (adjusted OR 1.24, 95\% CI 1.11-1.3) (13). While assessing mortality trends over their study period from years 2005-2011, they found improved survival in the year 2011 compared to year 2005 (31\% vs. $21 \%, \mathrm{p}<0.001)$. In a more recent study from Get with the Guidelines Registry, Starke et al. evaluated 31,144 patients who suffered in-hospital SCA and stratified outcomes based on dialysis status (14). After multivariate adjustment, they found similar odds of survival to discharge (adjusted OR 1.05, 95\% CI 0.97-1.13) and survival with a favorable neurologic status (adjusted OR 1.12, 95\% CI 1.04-1.22) in ESRD patients when compared to their non-ESRD counterparts. In our study, we also demonstrated similar mortality rates in ESRD and non-ESRD patients after in-hospital SCA in PS matched cohorts. Additionally, in our trend analysis, we have also shown improved mortality in both ESRD and non-ESRD patients after in-hospital SCA over our study years despite an initial spike in year 2009 (figure 3). American Heart Association (AHA)/Emergency Cardiovascular Care (ECC) CPR guidelines were updated in 2010 
and focused primarily on early chest compressions (chest compression-airway-breathing rather than airwaybreathing-chest compressions as recommended by earlier guidelines), chest compressions of at least 2 inches with a rate of at least 100/minute, eradication of atropine use for non-shockable SCA and prompt institution of targeted temperature management in eligible patients $(15,16)$. It is plausible that improved mortality trend witnessed in our study in both ESRD and non-ESRD patients especially after 2010 may be related to wider application of revised AHA/ECC guidelines across all patient sub-groups.

Our analysis showed mortality was in excess of $70 \%$ in ESRD patients who suffered in-hospital SCA. The first step in reducing this mortality in ESRD patients is to adequately identify risk factors that are associated with in-hospital SCA so that targeted risk modification can be done. Shastri et al. assessed 1745 dialysis patients from the noncardiac deaths in the hemodialysis (HEMO) study and found that prior history of diabetes, peripheral vascular disease and ischemic heart disease were independently associated with SCA events in dialysis patients (17). After incorporating these variables in a SCA prediction model, they found good discrimination (C-statistic of $0.75,95 \%$ CI $0.70-0.79$ ) and calibration of the model at 3 years of followup. Our study also showed increased prevalence of diabetes $(32.5 \%$ vs. $8.4 \%, \mathrm{p}<0.01)$, peripheral vascular disease $(19.9 \%$ vs. $9.9 \%, \mathrm{p}<0.01)$ and coronary artery disease $(39.4 \%$ vs. $30.8 \%, \mathrm{p}<0.01)$ in ESRD patients who sustained in-hospital SCA when compared to non-ESRD patients. Additionally, in our predictor analysis, both diabetes (OR 1.046, 95\% CI 1.009-1.084) and peripheral vascular disease (OR 1.079, 95\% CI 1.044-1.115) were associated with increased mortality after in-hospital SCA among ESRD patients.

The current data on therapeutic interventions that can prevent SCA or improve outcomes after a SCA event in ESRD patients are limited. In a randomized, placebo-controlled trial on 114 consecutive dialysis patients with history of dilated cardiomyopathy, carvedilol administration was associated with $24 \%$ reduction in mortality at two years and a trend towards reduced incidence of SCA (18). On the contrary, a secondary analysis of HEMO study did not show any benefit of beta-blocker utilization in reducing incidence of SCA (19). The utilization of calcium channel blockers of dihydropyridine class is associated with improved survival at 24 hours after an index SCA event (20). Implantable cardioverter defibrillators have been shown to improve outcomes when utilized for secondary prevention purposes, however, they are often underutilized in ESRD patients due to multitude of factors (21). Additionally, dialysis prescription offers several opportunities to reduce risk of SCA among ESRD patients. Large fluctuations in serum electrolytes and fluids have been demonstrated as inciting factors for initiation of SCA in ESRD patients. Low potassium and calcium dialysates are especially associated with increased SCA as they increase the risk of hypokalemia and hypocalcemia during a dialysis session that disperses myocardial repolarization which is a well-recognized prerequisite for initiation of malignant arrhythmias $(5,22,23)$. Our data, unfortunately, do not inform on these patient and dialysis related characteristics. However, prompt attention to these measures can result in prevention of SCA events in ESRD patients and result in improved outcomes after such events have occurred.

\section{Limitations:}

Our study results should be interpreted in the context of following key limitations. (1) NIS is an administrative, claims based database that relies on ICD coding system. These codes can be subjected to errors, however, HCUP quality control measures are routinely instituted that mitigate such concerns (9). Additionally, the positive predictive value of ESRD codes is close to $96 \%(24)$. (2) There are no specific ICD codes for in-hospital arrest and previous studies have utilized demonstration of CPR as evidence of in-hospital arrest and we have replicated the same methodology in our current analysis. (3) NIS does not inform on detailed management of SCA and specifically no data is collected on quality of CPR and other measures that are practiced as part of advanced life support. (4) NIS censors data gathering at discharge and patients are not followed longitudinally. ESRD patients who sustained SCA have been shown to have poor survival on follow-up studies and that cannot be investigated from NIS dataset (25).

\section{Conclusion:}

In our large nationally representative sample of in-hospital SCA patients, we have shown that in adjusted analysis, inpatient mortality is similar in ESRD and non-ESRD patients. ESRD patients who sustained an 
in-hospital SCA have higher burden of key co-morbidities. Mortality has been on the downtrend trend after an in-hospital SCA event in both ESRD and non-ESRD patients over our study years.

\section{References:}

1. Saran R, Li Y, Robinson B, Ayanian J, Balkrishnan R, Bragg-Gresham J, Chen JT, Cope E, Gipson D, He K, Herman W, Heung M, Hirth RA, Jacobsen SS, Kalantar-Zadeh K, Kovesdy CP, Leichtman AB, Lu Y, Molnar MZ, Morgenstern H, Nallamothu B, O'Hare AM, Pisoni R, Plattner B, Port FK, Rao P, Rhee CM, Schaubel DE, Selewski DT, Shahinian V, Sim JJ, Song P, Streja E, Kurella Tamura M, Tentori F, Eggers PW, Agodoa LY, Abbott KC. US Renal Data System 2014 Annual Data Report: Epidemiology of Kidney Disease in the United States. Am J Kidney Dis. 2015;66(1 Suppl 1):Svii, S1-305

2. Alqahtani F, Almustafa A, Shah K, Akram Y, Abbasi D, Rattan R, Subrayappa N, Alkhouli M, Munir MB. Sudden cardiac arrest in end-stage renal disease patients on dialysis: A nationwide study. Pacing Clin Electrophysiol. 2018;41:1467-1475

3. HaiderAW, Larson MG, Benjamin EJ, LevyD. Increased left ventricular mass and hypertrophy are associated with increased risk for sudden death. J AmColl Cardiol. 1998;32:1454-1459

4. Campese VM. Left ventricular function and chronic kidney disease: How soon does it start? Nephrol Dial Transplant. 2014;29:1989-1991

5. Genovesi S, Dossi C, Viganò MR, Galbiati E, Prolo F, Stella A. Electrolyte concentration during haemodialysis and QT interval prolongation in uraemic patients. Europace. 2008;6:771-777

6. Karaboyas A, Zee J, Brunelli SM, Usvyat LA, Weiner DE, Maddux FW, Nissenson AR, Jadoul M, Locatelli F, Winkelmayer WC, Port FK, Robinson BM, Tentori F. Dialysate Potassium, Serum Potassium, Mortality, and Arrhythmia Events in Hemodialysis: Results From the Dialysis Outcomes and Practice Patterns Study (DOPPS). Am J Kidney Dis. 2017;69:266-277

7. Mix TC, St peter WL, Ebben J, Xue J, Pereira BJ, Kausz AT, Collins AJ. Hospitalization during advancing chronic kidney disease. Am J Kidney Dis. 2003;42:972-81

8. Girotra S, Nallamothu BK, Spertus JA, Li Y, Krumholz HM, Chan PS; American Heart Association Get with the Guidelines-Resuscitation Investigators. Trends in survival after in-hospital cardiac arrest. N Engl J Med. 2012;367:1912-1920

9. Agency for Healthcare Research and Quality, Rockville, MD. http://www.hcup-us.ahrq.gov/nisoverview.jsp. Accessed March 25, 2020

10. Kolte D, Khera S, Aronow WS, Palaniswamy C, Mujib M, Ahn C, Iwai S, Jain D, Sule S, Ahmed A, Cooper HA, Frishman WH, Bhatt DL, Panza JA, Fonarow GC. Regional

variation in the incidence and outcomes of in-hospital cardiac arrest in the United

States. Circulation. 2015;131:1415-25

11. Morita Y, Haruna T, Haruna Y, Nakane E, Yamaji Y, Hayashi H, Hanyu M, Inoko M. Incidence, predictors, causes, and costs of 30-day readmission after in-hospital cardiopulmonary resuscitation in the United States. Resuscitation. 2019;134:19-25

12. Andersen LW, Holmberg MJ, Berg KM, Donnino MW, Granfeldt A. In-Hospital Cardiac Arrest: A Review. JAMA. 2019;321:1200-1210

13. Saeed F, Adil MM, Malik AA, Schold JD, Holley JL. Outcomes of In-Hospital Cardiopulmonary Resuscitation in Maintenance Dialysis Patients. J Am Soc Nephrol. 2015;26:3093-101

14. Starks MA, Wu J, Peterson ED, Stafford JA, Matsouaka RA, Boulware LE, Svetkey LP, Chan PS, Pun PH. In-Hospital Cardiac Arrest Resuscitation Practices and Outcomes in Maintenance Dialysis Patients. 
Clin J Am Soc Nephrol. 2020;15:219-227

15. Field JM, Hazinski MF, Sayre MR, Chameides L, Schexnayder SM, Hemphill R, Samson RA, Kattwinkel J, Berg RA, Bhanji F, et al. Part 1: executive summary: 2010 American Heart Association guidelines for cardiopulmonary resuscitation and emergency cardiovascular care. Circulation. 2010;122(18 suppl 3):S640S656

16. Travers AH, Rea TD, Bobrow BJ, Edelson DP, Berg RA, Sayre MR, Berg MD, Chameides L, O'Connor RE, Swor RA. Part 4: CPR overview: 2010 American Heart Association guidelines for cardiopulmonary resuscitation and Emergency Cardiovascular Care. Circulation. 2010;122(18 suppl 3):S676-S684

17. Shastri S, Tangri N, Tighiouart H, Beck GJ, Vlagopoulos P, Ornt D, Eknoyan G, Kusek JW, Herzog C, Cheung AK, Sarnak MJ. Predictors of sudden cardiac death: a competing risk approach in the hemodialysis study. Clin J Am Soc Nephrol. 2012;7:123-30

18. Cice G, Ferrara L, D'Andrea A, D'Isa S, Di Benedetto A, Cittadini A, Russo PE, Golino P, Calabrò R. Carvedilol increases two-year survivalin dialysis patients with dilated cardiomyopathy: a prospective, placebo-controlled trial. J Am Coll Cardiol. 2003;41:1438-44

19. Tangri N, Shastri S, Tighiouart H, Beck GJ, Cheung AK, Eknoyan G, Sarnak MJ. $\beta$-Blockers for prevention of sudden cardiac death in patients on hemodialysis: a propensity score analysis of the HEMO Study. Am J Kidney Dis. 2011;58:939-45

20. Pun PH, Lehrich RW, Smith SR, Middleton JP. Predictors of survival after cardiac arrest in outpatient hemodialysis clinics. Clin J Am Soc Nephrol. 2007;2:491-500

21. Munir MB, Alqahtani F, Aljohani S, Bhirud A, Modi S, Alkhouli M. Trends and predictors of implantable cardioverter defibrillator implantation after sudden cardiac arrest: Insight from the national inpatient sample. Pacing Clin Electrophysiol. 2018;41:229-237

22. Pun PH, Lehrich RW, Honeycutt EF, Herzog CA, Middleton JP. Modifiable risk factors associated with sudden cardiac arrest within hemodialysis clinics. Kidney Int. 2011;79:218-27

23. Pun PH, Horton JR, Middleton JP. Dialysate calcium concentration and the risk of sudden cardiac arrest in hemodialysis patients. Clin J Am Soc Nephrol. 2013;8:797-803

24. Vlasschaert ME, Bejaimal SA, Hackam DG, Quinn R, Cuerden MS, Oliver MJ, Iansavichus A, Sultan N, Mills A, Garg AX: Validity of administrative database coding for kidney disease: a systematic review. Am J Kidney Dis. 2011;57:29-43

25. Pun PH, Lehrich RW, Smith SR, Middleton JP. Predictors of survival after cardiac arrest in outpatient hemodialysis clinics. Clin J Am Soc Nephrol. 2007;2:491-500

Table and figure legends:

Table 1: Unadjusted and adjusted baseline characteristics of the study population

Table 2: Table 2: Unadjusted and propensity score matched outcomes of the study population

Figure 1: Flow sheet of patient selection

Figure 2: Proportion of end stage renal disease and non-end stage renal disease patients with in-hospital sudden cardiac arrest over study years

Figure 3: Trends in mortality in end stage renal disease and non-end stage renal disease patients after in-hospital sudden cardiac arrest over the study years

Figure 4: Length of stay trends in end stage renal disease and non-end stage renal disease patients after in-hospital sudden cardiac arrest over study years

Figure 5: Predictors of mortality in end stage renal disease patients after in-hospital sudden cardiac arrest 


\section{Hosted file}

flow chart 1.docx available at https://authorea.com/users/315790/articles/446054-baseline-characteristicsand-outcomes-of-end-stage-renal-disease-patients-after-in-hospital-sudden-cardiac-arresta-national-perspective
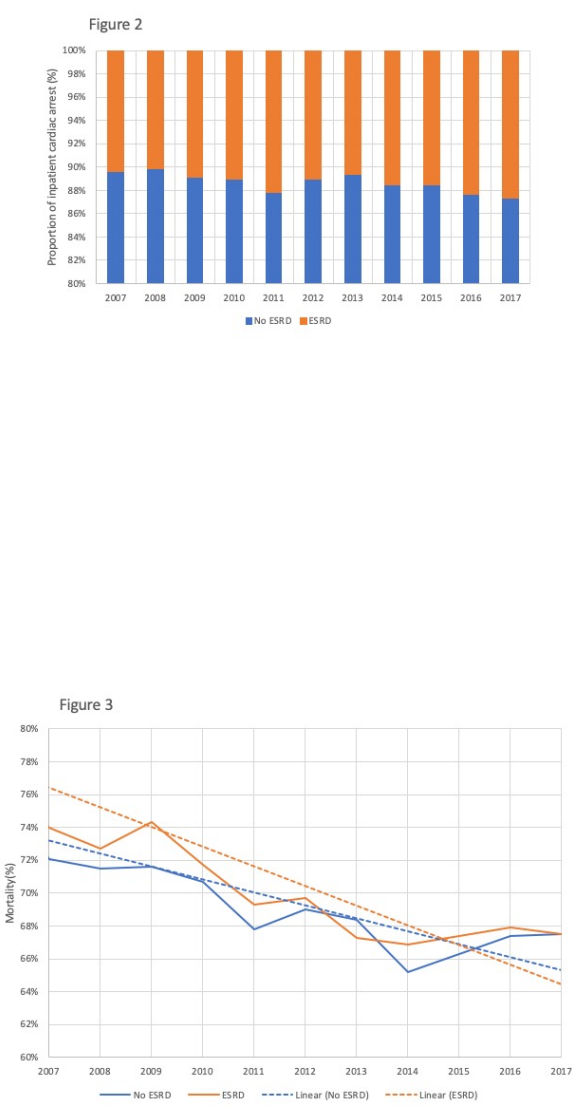

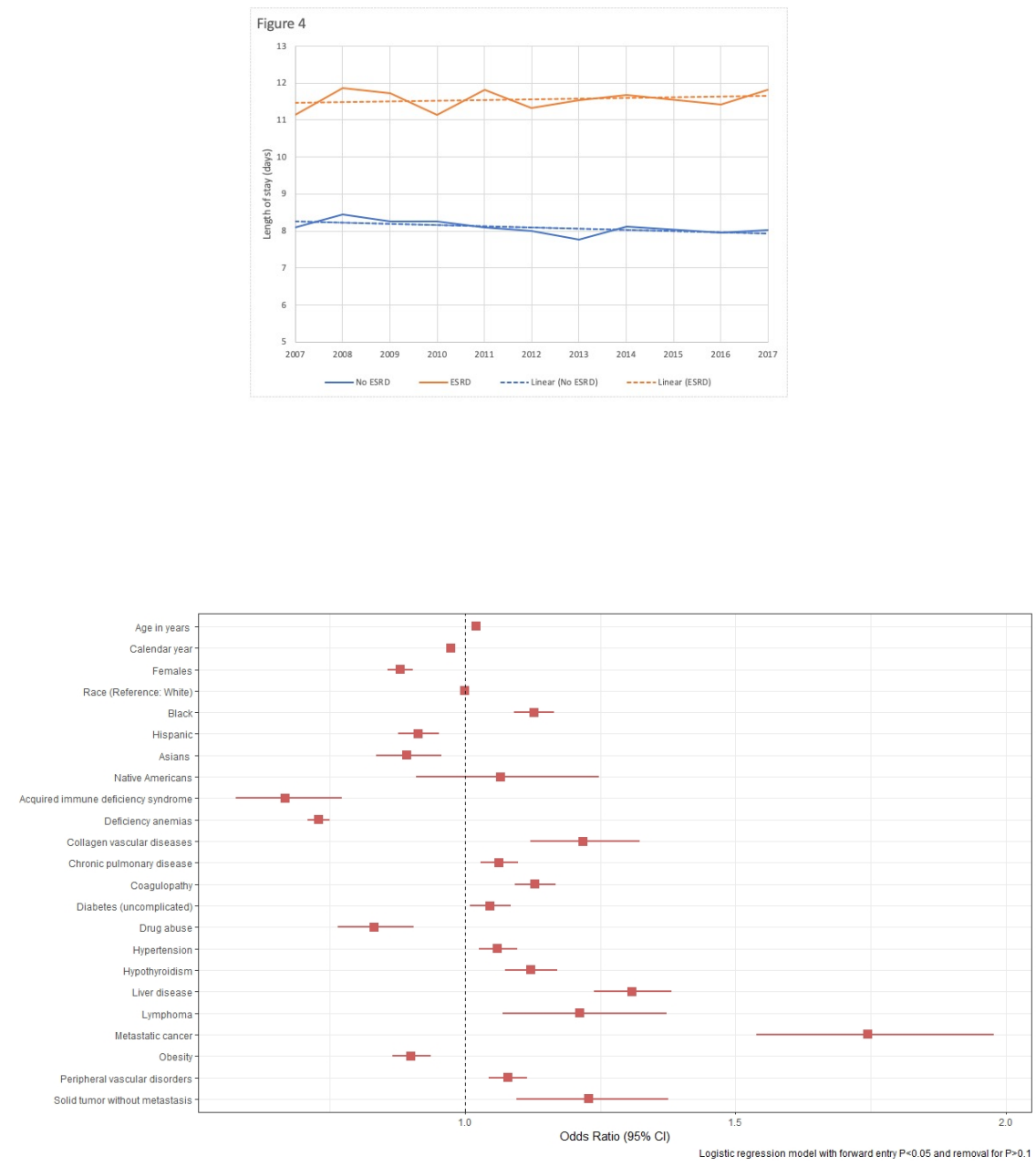